\title{
Multi-Classification and Automatic Text Summarization of Kannada News Articles
}

\author{
Anusha B. S. \\ Department of Computer Science \\ and Engineering \\ PES University, Bengaluru
}

\author{
Harshitha P. \\ Department of Computer Science \\ and Engineering \\ PES University, Bengaluru
}

\author{
Divya Ramesh \\ Department of Computer Science \\ and Engineering \\ PES University, Bengaluru
}

\author{
Uma D. \\ Department of Computer Science and Engineering \\ PES University, Bengaluru
}

\author{
Lalithnarayan C. \\ Electronics and Communication Engineering \\ PES University, Bengaluru
}

\begin{abstract}
Kannada is a historical language with abundant resources which display the tradition and culture of Karnataka. Extraction of most important and meaningful information from one or more large documents of text in the form of summary is a challenging task in regional languages compared to English. The main objective of present paper is to get the automatic summary of news articles from several sources. Naive-Bayes algorithm is used for classification of different categories of news articles include sports, politics and general. To find the sub-categories from each category such as state, national and international a Rock clustering algorithm has been used and the summary have been extracted automatically. Data is collected from multiple sources of summarization. A word vectorising stemmer approach is used to reduce the morphological complexity of the resources and a sub-sampling approach is used for efficient optimization and to reduce the complexity
\end{abstract}

\section{General Terms}

Natural Language Processing, Text Summarization, ROCK Clustering, Classification

\section{Keywords}

Text Summarization, Under Resourced Language, ROCK Clustering, Naïve- Bayes, Classification

\section{INTRODUCTION}

Increased interest in electronic media has enabled all languages to thrive well, and low resource languages have an opportunity to be prepared in terms of efficient techniques in the domain of Information Retrieval (IR), when the data grows sufficiently large. There is a great need to capture the salient details out of these materials which are unstructured in nature and the task of Automatic Text Summarization (ATS) comes into role. ATS is a technique or a process through which a text document is shortened to a concise summary, which reduces the time taken to understand documents. The focus of such summarization techniques is to find a subset of data which contains the meaningful information of the entire set in a precise manner. The need for it is realized through its usage. It helps in reducing reading time, summarizing research documents to make the selection process easier, to improve effectiveness of indexing, to get personalized summaries in question-answering system, to enable commercial abstract services to increase the number of texts they can process and many more. Availability of an enormous amount of textual material due to over-use of internet, comprised of web pages, news articles, status updates, blogs and so much more and it is growing every single day tremendously. The challenge here is to deal with unstructured data and extract meaningful textual information in the form of digital documents quickly accumulates to huge amounts of data.

The objective of this problem is to create an abstract of the entire news article or a representative summary by finding the most informative and relevant sentences. A lot of work has been done in summarization for English, but the work done on the same for Kannada is very less. Summarization of news articles from multiple sources has not been addressed so far. This kind of work might help decision makers of an organization when they need to make summaries from several sources. The same approach can be extended to images called as image summarization which finds the most representative and salient images. In case of surveillance videos, one can extract the important events from the uneventful context. Due to these reasons, ATS is a challenging open research problem and gaining more popularity among the researcher's group recently.

Kannada being a highly morphologically rich language makes it hard to apply NLP techniques. The need to automate evaluation of resources makes it important that the computer understand every word in the context. The text is transformed through a word vectorizing stemmer which reduces the morphological complexity of the resources. For purposes of generation of word embeddings and efficient optimization, sub sampling is performed. The word vectorizing stemmer improves the sub sampling process. The reduced complexity increases the efficiency of evaluation of texts.

\section{MORPHOLOGICAL COMPLEXITY AND ITS IMPLICATIONS}

The languages are characterized by the variety of words that are in use. Regional languages of India have very high degree of complex words, as these languages have evolved over ages, and many words have been borrowed from Sanskrit which again is a highly morphological language. The challenge with morphological complexity includes higher generalized algorithms and larger dataset to train on. Thus, reducing the morphological complexity is a key process in developing NLP algorithms.

Summarization is the task of finding relevant sentences in the document to simplify the process of information extraction for both humans and computers. In Kannada, the summarization 
process increases the impact the language has on the worldwide media, thus the summarization process is important. Difficulty in summarization process increases due to the morphological complexity of Kannada, thus producing inaccurate TF-IDF scores, which reduces the quality of summaries produced.

\subsection{Automatic Text Summarization}

There are two types of summarization that can be used for this task. They are, Extractive and Abstractive. Extractive methods work by selecting a subset of existing words, phrases, or sentences in the original text to form the summary. These models are based on statistical approaches, where the relevance of sentences is decided by a certain metric, for example, the TF-IDF score in our case. Work carried out till date has focused primarily on extractive methods, which are appropriate for image collection summarization and video summarization. In contrast, abstractive methods build an internal semantic representation and then use natural language generation techniques to create a summary that is closer to what a human might express. The internal representations are built using deep learning models, using Recurrent Neural Networks (RNN), Long Short Term Memory (LSTMs) and Gated Recurrent Units(GRUs).

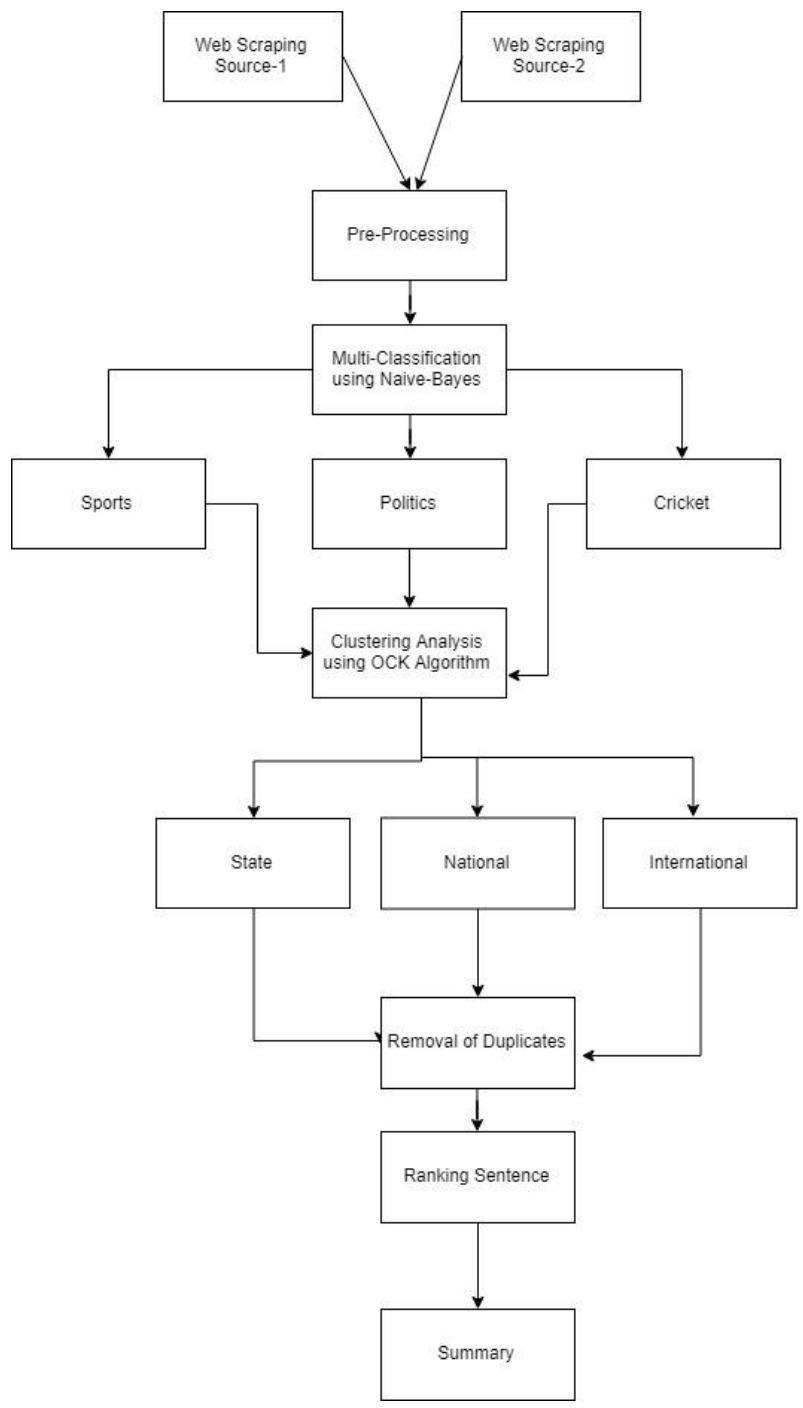

Fig. 1: Block Diagram

\section{LITERATURE SURVEY}

The work conducted in this domain is particularly on English. When it comes to Kannada, the research is limited, thus necessitating the need to implement the Natural Language Processing (NLP) techniques along with modifications to handle the morphological complexity. TF-IDF approach has been considered by [1] for text summarization. The results have been classified into various classes, based on Galavotti, Sebastiani, Simi (GSS) scores. They reported preprocessing techniques and impact of preprocessing techniques on summary outputs. Much of the work has been done in English, whereas, [2] implemented a system which considers different scoring mechanisms, like sentence based, word based and graph-based mechanisms, and then chooses sentences that best represent the summary. Fuzzy approach is considered in [3] using a TF-ISF (Inverse Sentence Frequency) approach. The semantic similarity between sentences and order of sentences are considered. [4] Implements a clustering-based approach to summarization, where sentences with high intra-class similarity are included in the summary. Similar classes are clustered in [5] provided the similarity is above a certain threshold derived from the ROCK clustering algorithm. Moreover, the ROCK algorithm considers even 'links' along with neighbors to cluster similar points together. Links are the number of common neighbors that exist between the two points. Global heap stores all these clusters, which is used to generate a 'goodness measure' for generating summaries. TF-IDF is used for clustering along with fuzzy k-means and hierarchical clustering algorithm in [6]. Machine Learning approach has been considered in [7], where the results suggest that larger datasets provide better results, which is a constraint in under resourced languages like Kannada.

\section{METHODOLOGY}

\subsection{Scraping}

The URL's of the RSS feeds and URL's of newspaper websites are used as input for downloading and parsing the news content for these sources. Initially the, the URL is parsed and then, content from the URL is extracted. Further, the content is parsed to obtain article title, article text and each article is output to a file.

\subsection{Preprocessing}

The articles that are scraped are scanned through to remove stop words. This is carried out as the results indicated by [8] improve drastically upon preprocessing. The content of the article is matched against a pre-generated set of stop words. The articles that have the stop words removed are given as input to the stemming algorithm. The stemming algorithm used is a morphological stemmer [9] the algorithm uses rules of morphology and improves the stemming accuracy by analyzing the process of agglutination in Kannada. The morphological complexity of Kannada prevents direct suffix stripping as cited in [10]. The stemmer works efficiently on the concept of sub-sampling.

\subsubsection{Sub-Sampling}

The stemmer reduces the variation in words by stripping the suffixes and reducing the number of unique words. By only selecting words that have a frequency higher than a threshold frequency, the process of optimization is improved. Thus, ignoring the terms with lower frequencies, and reducing the size of the dataset creates a smaller subset with more accurate information. Thus, creating summaries based on these terms that have been stemmed, increases the TF score for each 
sentence thereby improving the accuracy of the summary produced

\subsection{Multi-Classification}

Keeping users interests in mind the news articles are classified as sports, politics, or general. To achieve this multiclassification Naive-Bayes algorithm has been used. Based on the popular Bayes probability theorem, this is a supervised probabilistic approach, in which, the idea is based on the fact that classification is the task of training a model based on labeled training data. The hypothesis function trained on the training data can be used to test on new data-points. The category of each article is determined using the training documents present in each of the categories. Vocabulary is obtained by collecting tokens (unigram and bigram) from all train data. Next step is to find the frequency of each token for each category. The number of words per each class in train data is obtained. For each word in training documents pertaining to each category, the words - likelihood is calculated. Laplace smoothing technique is applied to avoid zero - results for unknown tokens. For each article that was scraped and is to be categorized, each token is extracted and using Bayes formula, the probability of the article belonging to each of the categories is calculated. The category with the highest probability is assumed to be the best suited category for that article. The article classified is mapped and stored to the folder named after that category.

\subsection{Clustering}

The categorized articles are taken as input to this module. The algorithm used to cluster the articles is the ROCK hierarchical clustering algorithm. The cosine similarity values are calculated for the documents and two documents that have a cosine similarity value above a certain threshold are said to be neighbors. The number of links for each document pair is then calculated where links represent the number of common neighbors between the pair of documents. Based on the link values the documents are clustered together. Clustered articles are given as the output from this module. The news articles in each category are clustered together based on the idea which is present in the articles so that redundant information is not presented to the user. The cosine similarity between documents is used as distance measure and the 'neighbors' and 'links' list of the documents are calculated based on the cosine similarity values. Using the 'neighbors' and 'links' list the ROCK clustering algorithm clusters similar news articles together.

\subsubsection{ROCK Clustering Algorithm}

ROCK is an iterative hierarchical clustering algorithm that uses the concepts of Neighbors and Links to cluster elements together. A point is said to be a Neighbor to another point if it satisfies the threshold condition. Each Neighbor of a point represents a single link value for that point. In addition to this the algorithm even has a local heap and a global heap that helps in clustering. Local heap has entries for each cluster which basically has the list of clusters that have link value greater than 0 for that cluster. Global heap has a list of all the clusters present. The algorithm uses ROCK to cluster documents that represent similar ideas into a single cluster. The sentences are scored using the tf-idf scoring measure. Then the cosine similarity between the documents is calculated to find how similar the documents are to each other. Two documents that have a cosine similarity greater than 0.6 are neighbors of each other. Neighbors list and Links list are then calculated for all the clusters. The local heap and global are constructed using the Neighbors list and Links list.
The first cluster is taken from the global heap and clustered with the first cluster in the local heap for \#those clusters are clustered together. The Links list, local heap and global heap are updated after the clustering. This step is repeated until no clusters have a local heap list of length more than 0 . The clusters obtained after this step is the final list of clusters obtained from the algorithm.

\subsubsection{Why Not Other Algorithms for Clustering?}

K-means chooses the initial centroid point randomly and since the clustering accuracy depends on the initial choice of centroids the accuracy can be low if the chosen centroids are not proper. Even the traditional hierarchical clustering algorithms may not be an appropriate choice for clustering categorical data. In hierarchical clustering clusters which have closest centroids are clustered together but considering only the distance between centroids to cluster may not be highly efficient for categorical data. As the size of the cluster grows it becomes very difficult to distinguish two clusters based on few attributes.

\subsection{Assigning Scores and Ranking Sentences}

The categorized articles are taken as input to this module. The sentences are scored by summing up the TF-IDF scores of each of the words and the category specific score. Each word is assigned a score based on its frequency in the document and a higher score is assigned to it if it is among the category specific words too. Each category has a background article which is totally unrelated to that category. It helps eliminate non-category specific words from the article, thus allowing singling out the category specific words which are then assigned a higher score.

\subsection{Removal of Duplicates}

The similarity value, ranging from 0 (dissimilar) to 1 (same) is used as a measure to obtain the degree of similarity between two sentences. The similarity value is obtained from mathematical computation using Levenshtein Edit Distance Algorithm which measures differences between two words based on minimum number of single-character edits. For every article that is previously clustered and ranked, duplication of sentences beyond certain level, which is susceptible to have similar meaning must be avoided. In the iteration, two sentences are checked for similarity and if found to be similar beyond a measure, one of them is removed. Levenshtein edit distance is a measure of the similarity between two strings. The distance is the number of deletions, insertions, or substitutions required to transform one string into another. Using edit distance, a similarity value ranging from 0 to 1 is obtained for two sentences. Similarity value greater than 0.5 is assumed to indicate sentence with similar meaning and hence one of the two sentences is removed and repeated across all sentences.

\section{EVALUATION}

The results include classification of data into various classes and sub- classes. The classes are 'Sports', 'Political' and 'General'. Under sports the classes are 'Cricket', 'Football' and 'General Sports'. Under Political, the classes are 'State', 'National' and 'International'. Prevention of zero probability from the Naive Bayes algorithm is taken care by including Laplace smoothing. Naive Bayes works efficiently for multiclass classification for categorical input variables compared to numerical variables. 


\subsection{Dataset}

The dataset retrieved from web scraping utilized various sources. The source of the dataset plays an important role in the generation of summary. The data used for the following were scraped from the following URL's.

1. rss:https://kannada.oneindia.com/rss/kannada-newsfb.xml

2. rss:http://www.kannadaprabha.com/rss/kannadanation $4 . \mathrm{xml}$

3. rss:http://www.kannadaprabha.com/rss/kannadasports7.xml

4. rss:http://www.kannadaprabha.com/rss/kannadacricket8.xml

\section{RESULTS}

News articles from different websites have been scraped for a day and multi-classification have been done to categorize them into different categories and clustering has been applied to make subcategories. Finally, summaries are extracted with respect to categories "General", "Sports", and "Political" after removal of duplication. The same results are shown in figures 3 to 6 . Figure 3 shows the summary of general news from all the above said articles. In addition to this, national level general news is shown in figure 4. Similarly, the general news about sports category can be found in figure 5 and a specific sub-category of sports such as cricket news are shown in figure 6 . The reason for choosing cricket as one of the main sub-categories of sport is due to the number of users to know about cricket related information would be more. This is not limited to only one sub-category. It can be extended to any number. This work helps the people who do not have time to read whole news articles but interested to know the information in short time without missing anything. This summary is not obtained from one news article, but from many due to the reason that different sources might be having some additional information.

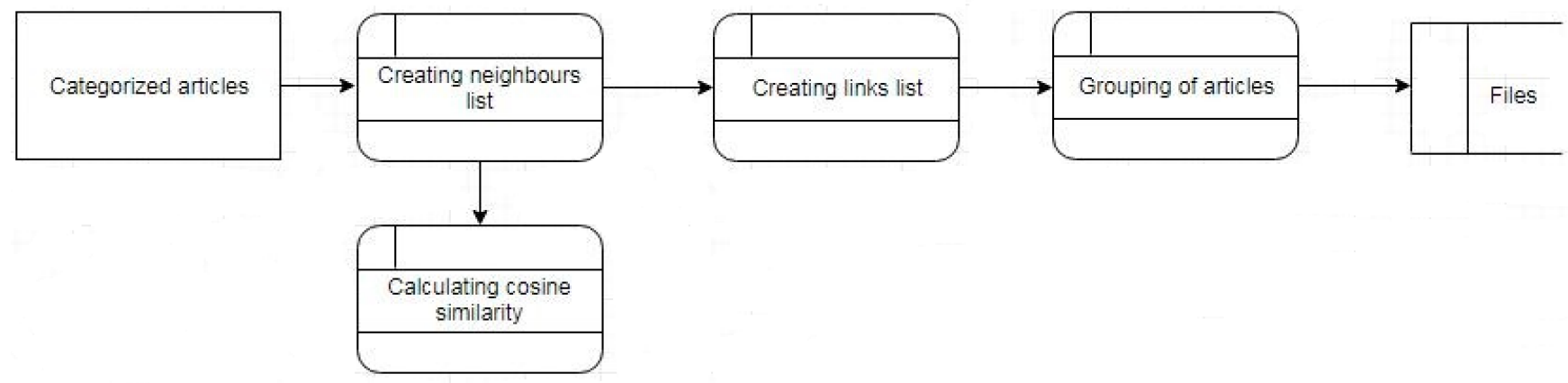

Fig. 2: Rock Clustering Algorithm

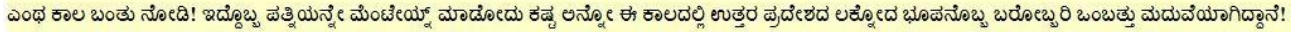

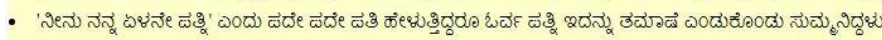

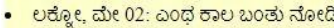

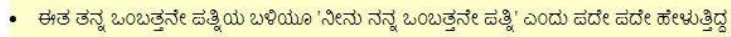

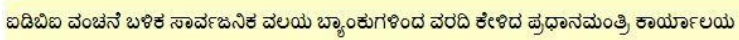

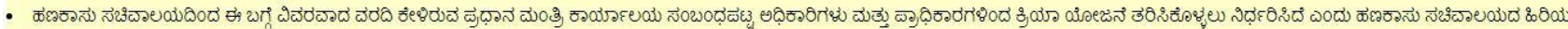

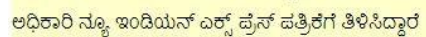

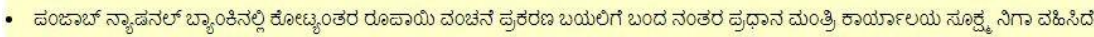

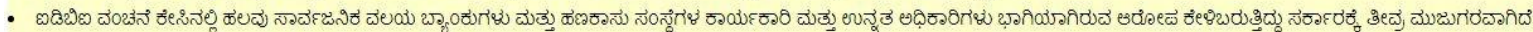

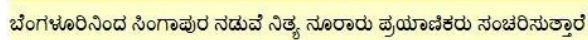

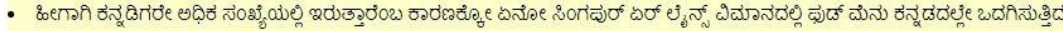

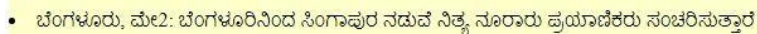

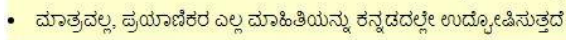

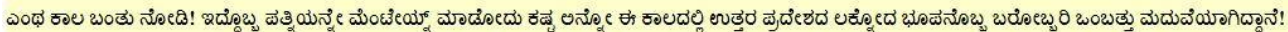

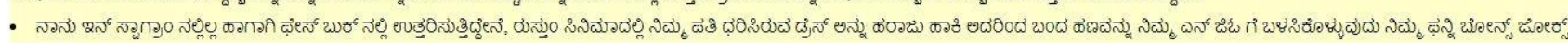

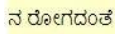

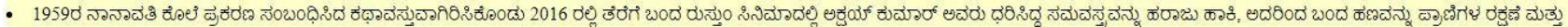

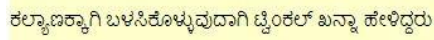

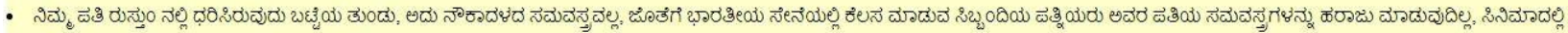

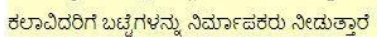

Fig. 3: Results: General News 


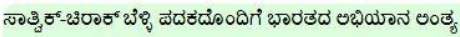

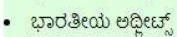

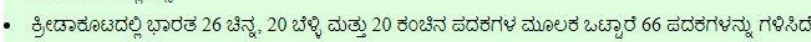

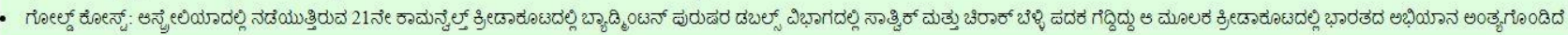

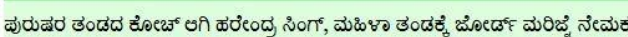

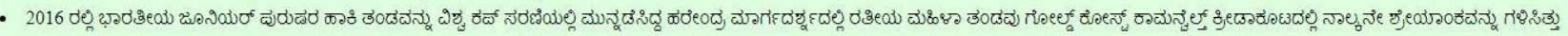

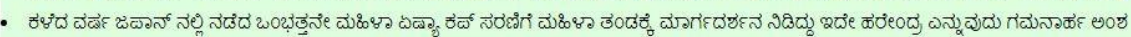

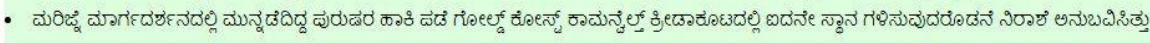

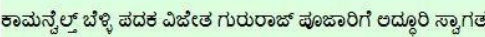

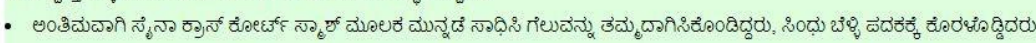

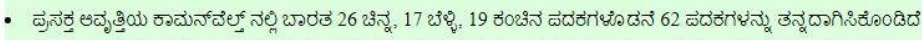

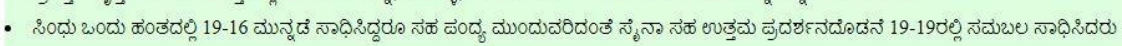

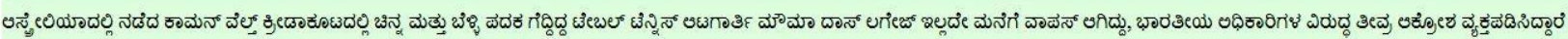

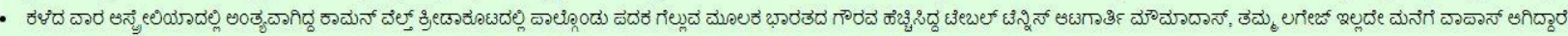

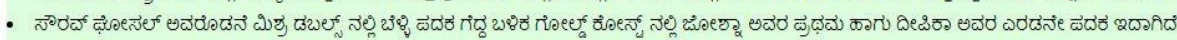

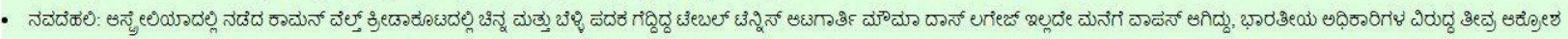
ద్యేర్తైడిడిసిద్వారి

\section{Fig. 4: Results: General Sports}

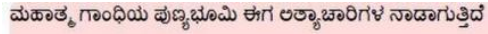

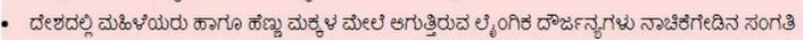

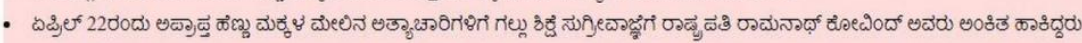

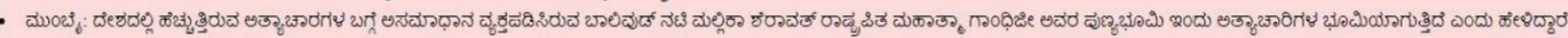

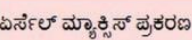

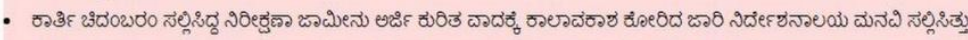

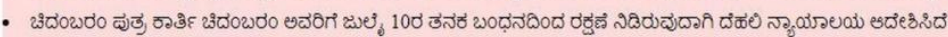

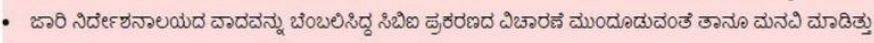

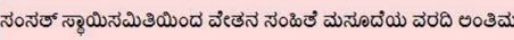

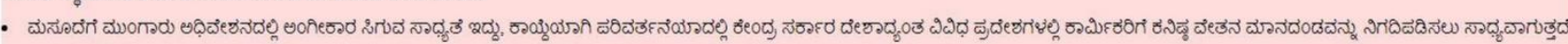

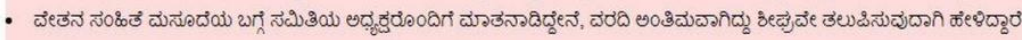

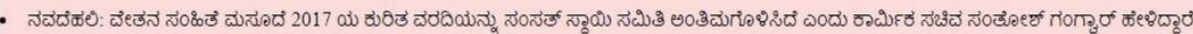

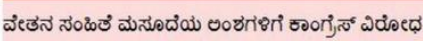

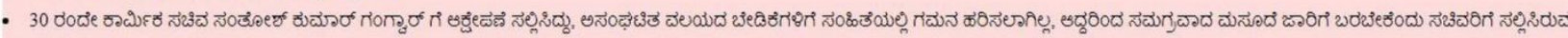

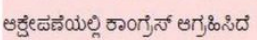

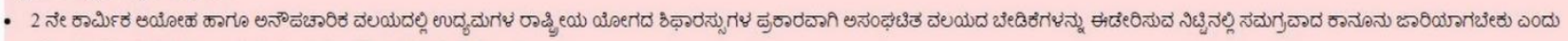

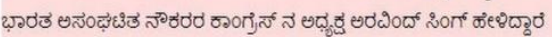

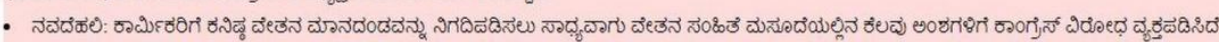

Fig. 5: Results: General News: National 


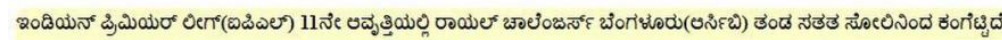

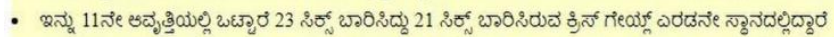

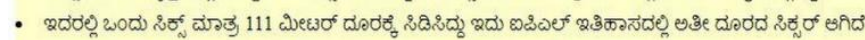

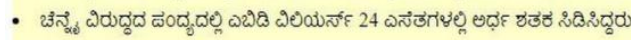

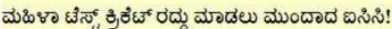

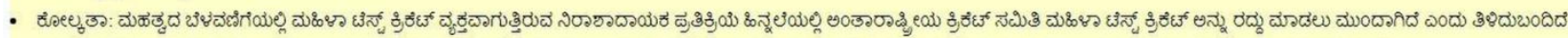

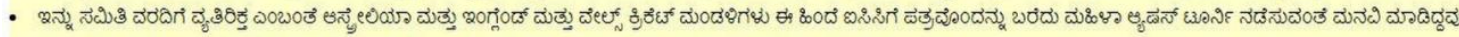

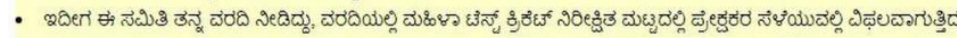

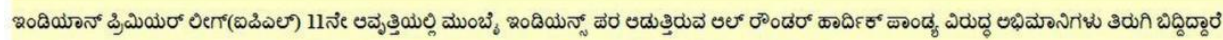

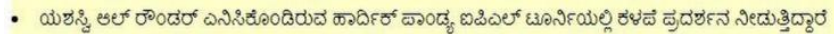

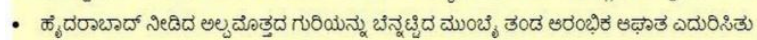

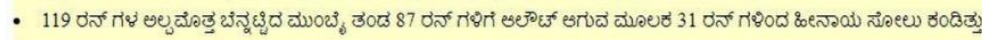

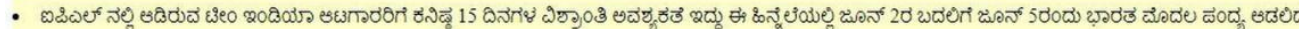

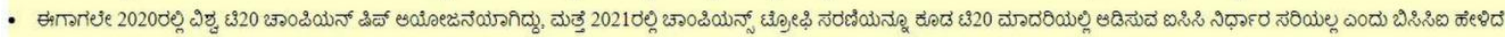

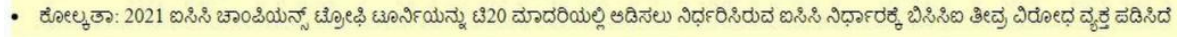

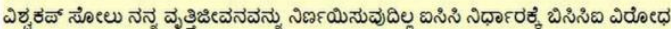

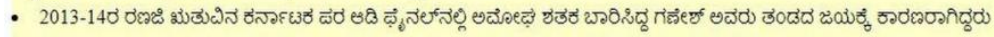

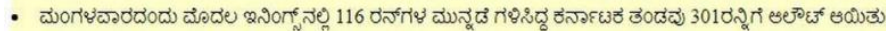

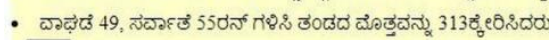

Start

\section{FUTURE WORK AND APPLICATIONS}

Text summarization in Kannada is a relatively new study, and not much work has been done in this field in Kannada. The application has taken only three categories into account Sports, Politics and General with Sports and Politics having three subcategories each. The work can be extended to include a greater number of categories so that the user gets more options to choose from. The application scrapes news articles from three websites only - Kannada Prabha, One India News and Sahil Online, and clusters similar articles by finding cosine similarity between sentences. This could be extended to include more sources and the articles could be combined by taking semantics into account which would improve the accuracy by quite a large amount. The application summarizes the content in an extractive manner, meaning, it does not take semantics into account while summarizing. It ranks the sentences based on various features and picks the sentences that best represent the theme of the content. Summarizing the articles by taking semantics into account can be another aspect for improvement.

\section{REFERENCES}

[1] Keyword extraction based summarization of categorized Kannada text documents, Jayashree, R and Murthy, Srikanta K and Sunny, K, International Journal on Soft Computing, Vol 2, Number 4, Pg- 81, Year, 2011

[2] A context based text summarization system, Ferreira, Rafael and Freitas, Frederico and de Souza Cabral, Luciano and Lins, Rafael Dueire and Lima, Rinaldo and França, Gabriel and Simske, Steven $J$ and Favaro, Luciano, 2014 11th IAPR International Workshop on Document Analysis Systems (DAS), pg- 66-70, 2014
[3] Automatic text summarization using fuzzy inference, Jafari, Mehdi and Wang, Jing and Qin, Yongrui and Gheisari, Mehdi and Shahabi, Amir Shahab and Tao, Xiaohui, Automation and Computing (ICAC), 2016 22nd International Conference on,pg- 256-260,2016

[4] Text summarization using Clustering technique, Deshpande, Anjali R and Lobo, LMRJ, International Journal of Engineering Trends and Technology

[5] ROCK: A robust clustering algorithm for categorical attributes, Guha, Sudipto and Rastogi, Rajeev and Shim, Kyuseok, Data Engineering, 1999. Proceedings., 15th International Conference on

[6] ] Document clustering: TF-IDF approach, Bafna, Prafulla and Pramod, Dhanya and Vaidya, Anagha, Electrical, Electronics, and Optimization Techniques (ICEEOT), International Conference on, Pg-61-66,2016

[7] Automatic text summarization using a machine learning approach, Neto, Joel Larocca and Freitas, Alex A and Kaestner, Celso AA, Brazilian Symposium on Artificial Intelligence

[8] An Improvised Extractive Approach to Hindi Text Summarization, Kumar, K Vimal and Yadav, Divakar, Information Systems Design and Intelligent Applications

[9] Multi-Level based Stemming Based on Rules of Morphology for the Purposes of Implementation of Word Embeddings on Kannada, Lalithnarayan C and Shylaja Sharath S S

[10] Kannada stemmer and its effect on Kannada documents classification, Deepamala, N and Kumar, P Ramakanth, Computational Intelligence in Data Mining-Volume 3 